\title{
LIDERAZGO DE LA ENFERMERA SUPERVISORA EN LA MOTIVACIÓN DEL PERSONAL DE ATENCIÓN DIRECTA EN UN HOSPITAL DE SEGUNDO NIVEL EN ICA, PERÚ
}

\begin{abstract}
LEADERSHIP OF THE SUPERVISOR NURSE IN THE MOTIVATION OF DIRECT CARE STAFF IN A HOSPITAL OF SECOND LEVEL IN ICA, PERÚ.
\end{abstract}

Recibido: 29 septiembre 2017 Aceptado: 22 diciembre 2017

Correspondencia: Dra. Olinda Oscco Torres Correo electrónico: olita_4@hotmail.com Dra. Lasty Balseiro Almario Correo electrónico: lastybals@hotmail.com

Palabras clave: enfermera supervisora, liderazgo, motivación.

Key words: supervisor nurse, leadership, motivation.

\section{Autores:}

Dra. Olinda Oscco Torres

Docente de la asignatura de Metodología de la Investigación UPSJB Filial Ica

Mg. Carmen E. Bendezú Sarcines

Docente de la asignatura de Elaboración de Proyectos de Investigación UPSJB Filial Ica

Mg. María L. Rojas Guillén

Docente de la asignatura de Enfermería en el Cuidado del Niño con Problemas de Salud UPSJB Filial lca

Lic. María L. Alejos Tasayco

Docente de la asignatura de Metodología de la Enseñanza UPSJB Filial Chincha

Dra. Lasty Balseiro Almario

Profesor de Carrera Titular B de la ENEO, adscrita a la Unidad de Investigación de la ENEO - UNAM, Ciudad de México 


\section{RESUMEN}

Objetivo: el objetivo de la investigación es determinar el estilo de liderazgo de las enfermeras supervisoras y su influencia en la motivación del personal de Enfermería de atención directa de un hospital de segundo nivel de la ciudad de lca, Perú.

Marco teórico: el liderazgo es el proceso de dirigir e influir en las actividades laborales de un grupo. La motivación es el estímulo que mueve a las personas a realizar determinadas acciones y persistir en ellos hasta el logro de objetivos.

Metodología: investigación cuantitativa, descriptiva, correlacional, explicativa y transversal. Las dimensiones de la variable independiente Estilo de Liderazgo fueron: democrático, autocrático y liberal y de la variable dependiente Motivación fueron intrínseca y extrínseca. La muestra estuvo constituida por 72 participantes seleccionados a través del muestreo probabilístico simple.

Resultados: el personal de enfermería refirió en un $51 \%$ estar altamente motivado extrínsecamente con el estilo de liderazgo, motivado en un 25\%, medianamente motivado en un 8\%, el 10\% poco motivado y el 6\% nada motivado. Respecto a la motivación intrínseca el 42\% está altamente motivada, 25\% motivada, 17\% medianamente motivada, 10\% poco motivada y 7\% nada motivada.

Discusión: los resultados reflejan que el personal de enfermería se encuentra motivado extrínseca e intrínsecamente con los tres estilos de liderazgo, lo cual podría deberse a que las enfermeras supervisoras convocan a reuniones para informar sobre las actividades a realizar, consultan ideas y opiniones, aceptando sus contribuciones siempre que sea posible y práctico. Conclusiones: Existe una relación positiva entre el estilo de liderazgo de las enfermeras supervisoras y la motivación del personal.

Palabras clave: enfermera supervisora, liderazgo, motivación.

\section{ABSTRACT}

Objective: the objective of the research is to determine the leadership style of the supervising nurses and their influence on the motivation of the direct care nursing staff of a second level hospital in the city of lca, Peru.

Theoretical framework: leadership is the process of directing and influencing the work activities of a group. Motivation is the stimulus that moves people to perform certain actions and persist in them until the achievement of goals.

Methodology: quantitative, descriptive, correlational, explanatory and transversal research. The dimensions of the independent variable Leadership Style were: democratic, autocratic and liberal and the dependent variable Motivation were intrinsic and extrinsic. The sample consisted of 72 participants selected through simple probabilistic sampling.

Results: nurses were $51 \%$ highly motivated by the style of leadership, motivated by 25\%, moderately motivated by $8 \%, 10 \%$ not motivated and $6 \%$ not motivated. Regarding the intrinsic motivation, 42\% is highly motivated, $25 \%$ motivated, $17 \%$ moderately motivated, 10\% not motivated and 7\% not motivated.

Discussion: the results reflect that nurses are motivated extrinsically and intrinsically with the three styles of leadership, which could be due to the Supervisor Nurses convening meetings to inform about the activities to be carried out, to consult ideas and opinions, accepting their Contributions where possible and practical. Conclusions: There is a positive relationship between the leadership style of the supervising nurses and the motivation of the staff.

Key words: supervisor nurse, leadership, motivation.

139 MMN/W4 Enf Neurol Vol. 16. No. 3 septiembre - diciembre 2017 


\section{INTRODUCCIÓN}

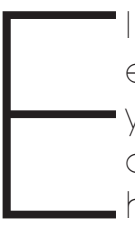

objetivo de la investigación es determinar el estilo de liderazgo de la enfermera supervisora

y su influencia en la motivación del personal de Enfermería de atención directa en un hospital de segundo nivel de lca, en el Perú. El problema formulado es ¿Cuál es la influencia del estilo del liderazgo de la enfermera supervisora, en la motivación del personal de enfermería de atención directa, en un hospital de segundo nivel de la ciudad de lca, en el Perú? La importancia de la investigación radica en fortalecer el campo del conocimiento y servir de insumo para otras investigaciones que aporten a la construcción permanente del concepto de gestión de liderazgo y motivación.

Según Díaz B.? el Liderazgo es el proceso de dirigir e influir en las actividades laborales de un grupo. Román J. y Ferrández M., ${ }^{2}$ refieren que la motivación es un asunto fundamental para el desarrollo ya que se traduce en crecimiento tanto individual como colectivo, dando como resultado el cumplimiento de los objetivos trazados lo cual, a su vez, se convertirá en motivación para los integrantes de los grupos de trabajo, que puede transformarse en liderazgo. Así, las personas que conforman un grupo de trabajo deben identificarse con un ideal común para poder sentirse a gusto y lograr los objetivos proyectados, además, debe haber una perfecta interacción entre los factores, equipo-tarea-individuo para poder atender las necesidades correspondientes a cada problema.

La motivación intrínseca en el trabajo viene dada por las características de la actividad laboral que se realiza, busca satisfacer necesidades superiores derivadas de la actividad laboral en sí misma y con control interno. La motivación extrínseca proviene del exterior, donde la actividad del sujeto es un medio hacia un fin. Esta actividad puede ser un medio para ganar dinero, fama y estatus. Este tipo de motivación centra a las personas en las metas y no en las vías para alcanzarlas.
Para Gonzales A, y Pérez S, el liderazgo de Enfermería necesita de una especial transmisión de poder e influencia que consiga dirigir el rumbo de profesionales en la dirección correcta, así como también de la capacidad de influir sobre el grupo para provocar el convencimiento de los valores, objetivos y estrategias corporativas compartidas. Laurencio $\mathrm{M}$, et al., ${ }^{4}$ manifiestan que, al invertir en el poder existente en los liderados, el líder rearticula ese poder en sintonía con el suyo para conseguir una alianza grupal en relación a objetivos comunes, manteniendo su influencia a través del refuerzo y del compromiso con ideales comunes. De igual forma para Amestoy S. et al., explica que, en el caso de enfermería, si el enfermero es responsable, comprometido y puntual, él podrá influir en su equipo para desarrollar esas mismas características.

Según Díaz M, et al., ${ }^{\circ}$ el liderazgo es un tema crucial hoy en día, en donde las fronteras se han abierto al comercio global, en el cual las organizaciones y empresas permanentemente se encuentran en una constante lucha por ser cada vez más competitivas, lo que ha generado que las personas que la conforman sean eficientes y capaces de dar mucho de sí para el bienestar de la organización o empresa. Bass B., señala que, en primer término el liderazgo involucra a otras personas, como los empleados o seguidores; en segundo término el liderazgo entraña una distribución desigual del poder entre los líderes y los miembros del grupo; el tercer aspecto del liderazgo es la capacidad para utilizar las diferentes formas del poder para influir en la conducta de los seguidores de diferentes maneras y, el cuarto aspecto, es una combinación de los tres primeros pero reconoce que el liderazgo es una cuestión de valores.

Díaz Z, y Escobar D. refieren que, aunque el liderazgo guarda una gran relación con las actividades administrativas y el primero es muy importante para la segunda, el concepto de liderazgo no es igual al de administración. Goleman D." ${ }^{9}$ manifiesta que, en muchos sentidos, el estilo de su líder define a una organización. Si la organización es fiel 
a su filosofía y misión, el estilo de su líder debe ser coherente con ellas. Un líder autocrático en una organización democrática puede crear el caos, un líder preocupado solo en el nivel administrativo de una organización basada en la importancia de los valores humanos, puede menguar el objetivo de su labor.

Balderas S., ${ }^{10}$ menciona la importancia de señalar que el supervisor se encuentra en una situación de superioridad jerárquica, ya que tiene la facultad o capacidad de determinar si la acción es correcta o no, la supervisión en enfermería es una de las funciones principales de gestión, liderazgo, dirección y control que realiza la enfermera para mejorar las condiciones de trabajo y garantizar una atención de calidad. El propósito de la supervisión en enfermería es promover el trabajo en equipo y desarrollar la eficiencia del personal para lograr la máxima productividad. La finalidad de la supervisión en enfermería es mejorar la calidad de atención, crear un ambiente favorable a las personas, impulsar el desarrollo del personal, mantener la disciplina y el interés en el trabajo y organizar la utilización de los recursos materiales. La distribución del tiempo que se dedica para la supervisión es un 45\% dirigida al personal, 20\% al paciente, 20\% al servicio, 10\% a la docencia y $5 \%$ a otras actividades.

Para Martínez P, y Fernández A.," la motivación se refiere a un proceso adaptativo, que es el resultado de un estado interno de un organismo, que le impulsa y le dirige hacia una acción en un sentido determinado. Es decir, es influenciado por factores externos e internos que activan el organismo y dirigen su conducta hacia la consecución de objetivos y metas gratificantes.

Dentro de la teoría humanista de Rosemarie Rizzo Parse, mencionada por Manrique F." ${ }^{12}$ Marriner-Tomey A, enfoca el descubrimiento del significado de las experiencias que han vivido las enfermeras respecto a la supervisión como parte de la administración; asimismo Bandura $\mathrm{A}_{.13}{ }^{13}$ dice que los empleados obtienen información fundamental acerca de cómo desempeñarse y comportarse por medio de la observación e imitación de los modelos a su alrededor.
Maxwell J.14 menciona que la motivación es un asunto fundamental para el desarrollo, ya que se traduce en desarrollo tanto individual como colectivo, dando como resultado el cumplimiento de los objetivos trazados. Guillen C.." ${ }^{15}$ refiere que se han dado muchas explicaciones sobre la motivación laboral, sobre las variables que motivan a las personas a llevar a cabo una tarea.

\section{METODOLOGÍA}

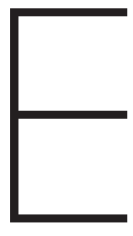
I estudio fue de tipo descriptivo correlacional, explicativo y transversal. La técnica para la recolección de datos fue la encuesta para ambas variables, para la variable estilos de liderazgo se aplicó el test de liderazgo de Kurt Lewin el cual consta de 33 reactivos correspondiente a tres dimensiones: autocrático, democrático y liberal, cuyas opciones de respuesta fueron "de acuerdo" y "en desacuerdo"; para la variable motivación se utilizó un test tipo escala de Likert, el cual fue sometido a validación de contenido por expertos en el área, con base en las sugerencias se corrigió el instrumento, quedando finalmente con veinte reactivos, divididos en dos dimensiones, con cinco alternativas de respuesta: "altamente motivada", "motivada", "medianamente motivada", "poco motivada" y "nada motivada".

La población estuvo constituida por 92 enfermeras y enfermerosy 130 técnicasytécnicos de enfermería de los servicios de hospitalización y consultorios externos; luego de aplicar la fórmula para población finita, la muestra definitiva fue de 72 participantes. Criterios de inclusión: enfermeros y técnicos de enfermería de hospitalización y consultorios externos, por su parte, en los criterios de exclusión se contempló al personal profesional de enfermería que realiza actividades administrativas, y los criterios de eliminación se aplicaron a aquellos que no desearon participar en la investigación. Los métodos estadísticos para el procesamiento y análisis de la información fueron; media aritmética para datos agrupados, desviación estándar muestral para datos agrupados y método estadístico de la prueba de Chi cuadrada.

\section{1} MMN Why 
Para recolectar los datos de la variable motivación, se utilizó un cuestionario tipo escala de Lickert elaborado por la autora, según los objetivos de la investigación. El cuestionario fue previamente validado a través del juicio de tres profesionales expertos, con grado de doctor especialistas en la materia. La prueba piloto quedó conformado por dos dimensiones, extrínseca e intrínseca, quedando constituida de 20 items con respuestas cerradas tipo escala de Likert con cinco alternativas. Las consideraciones éticas de beneficencia, no malefi-cencia, respeto y autonomía, se mantuvieron durante todo el proceso de la investigación. También se consideró el consentimiento informado de los participantes, remarcando que su participación en la investigación es confidencial y no ocasionará ningún perjuicio a su persona.

\section{RESULTADOS}

$R$ especto a los datos generales se encontró que el 57\% tienen de 41 a 56 años; el sexo predominante es el femenino en el 80\%; un 47\% es de estado civil casado, el 75\% tiene de 12 años a más de experiencia laboral y el 67\% son de condición laboral (ver tabla No. 1). En relación a la motivación extrínseca, el personal de enfermería refirió estar altamente motivado con el estilo de mando democrático en un 28\%, con el estilo autocrático 19\% y con el estilo liberal solo el 4\%. Asimismo, un 14\% refirió estar motivado con el estilo democrático, el 8\% con el estilo autocrático y un 3\% con el estilo liberal, el 4\% refiere estar medianamente motivada con el estilo democrático, el 3\% con el estilo liberal y el 1\% con el estilo autocrático. También el 7\% refiere estar poco motivada con el estilo liberal, el 3\% con el estilo autocrático y nada motivada el 4\% con el estilo liberal y el 1\% con el estilo autocrático (ver tabla No. 2).

Tabla 1. Datos sociodemográficos del personal de enfermería de atención directa en un hospital de segundo nivel, lca - Perú

\begin{tabular}{|c|c|c|c|c|}
\hline EDAD & & FR & & $\%$ \\
\hline De 25 a 40 años & 1 & 25 & ' & 35 \\
\hline De 41 a 56 años & i & 41 & ; & 57 \\
\hline De 57 años a más & I & 6 & , & 8 \\
\hline \multicolumn{5}{|l|}{ SEXO } \\
\hline Masculino & ' & 8 & i & 11 \\
\hline Femenino & ' & 64 & ' & 89 \\
\hline \multicolumn{5}{|l|}{ ESTADO CIVIL } \\
\hline Soltera & , & 26 & , & 36 \\
\hline Casada & ; & 34 & ; & 47 \\
\hline Conviviente & , & 10 & , & 14 \\
\hline Divorciada & ' & 2 & 1 & 3 \\
\hline \multicolumn{5}{|l|}{ EXPERIENCIA LABORAL } \\
\hline Menos de 5 años & , & 12 & i & 17 \\
\hline De 6 a 11 años & ; & 6 & ; & 8 \\
\hline De 12 años a más & ， & 54 & ; & 75 \\
\hline CONDICIÖN LABORAL & 1 & & 1 & \\
\hline Nombrado & i & 44 & ; & 61 \\
\hline Plaza orgánica & i & 18 & i & 25 \\
\hline Contrato cas & : & 10 & ' & 14 \\
\hline Total & 1 & 72 & ' & 100 \\
\hline
\end{tabular}

Fuente: Encuesta realizada al personal de enfermería de un hospital de segundo nivel de la ciudad de lca Perú, 2015. 
Tabla 2. Estilo de liderazgo de la enfermera supervisora relacionado a la motivación extrínseca del personal de enfermería de atención directa en un hospital de segundo nivel en lca - Perú

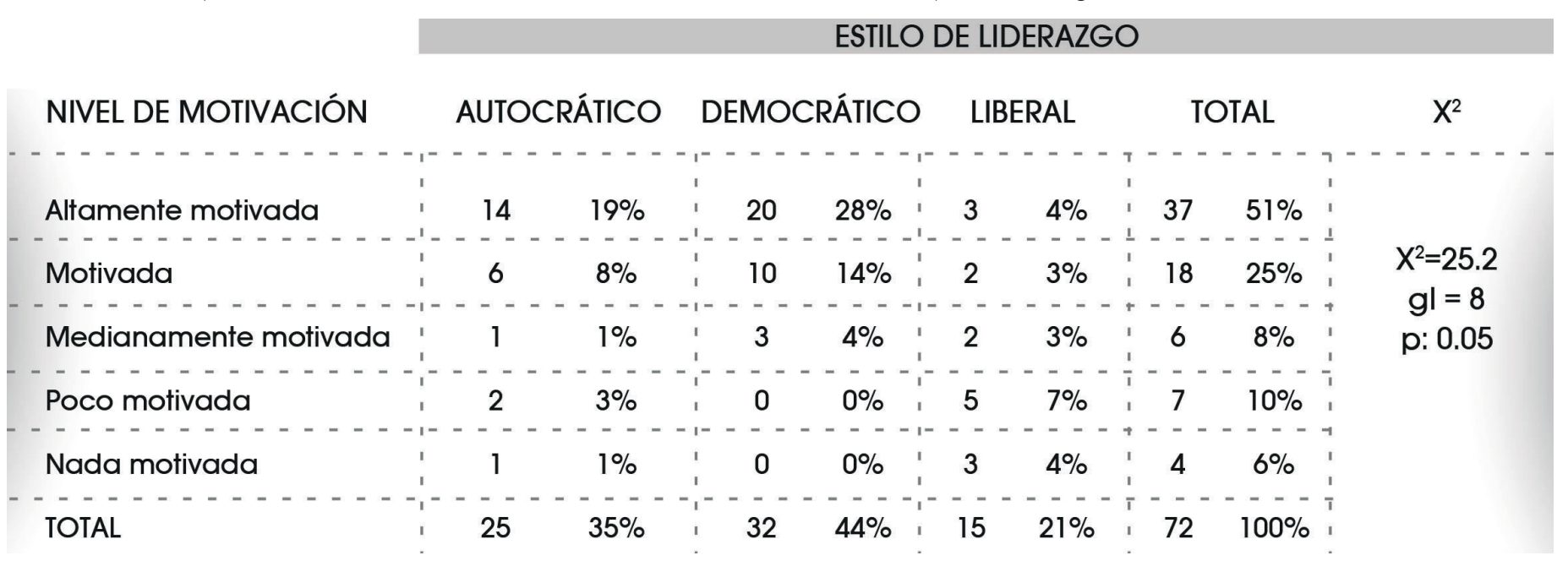

Fuente: Encuesta realizada al personal de enfermería de un hospital de segundo nivel de la ciudad de lca Perú, 2015.

En relación a la motivación intrínseca, el personal de enfermería refirió en un 22\% estar en gran medida motivado con el estilo de mando democrático; el 18\% con el estilo autocrático y el 1\% con el estilo liberal. De igual modo, un 15\% refirió estar motivado con el estilo democrático, el 7\% con el estilo autocrático y el 3\% con el estilo liberal; el 7\% refiere estar medianamente motivada con el estilo liberal, el 6\% con el estilo democrático y el 4\% con el estilo autocrático; el 6\% refiere estar poco motivada con el estilo liberal, el 3.\% con el estilo autocrático y el 1\% con el estilo democrático (ver tabla No.3). De esta forma, el personal se encuentra nada motivada en un 4\%, con el estilo liberal, el 1\% con el estilo democrático y con el estilo autocrático respectivamente.

Tabla 3. Estilo de liderazgo de la enfermera supervisora relacionado a la motivación intrínseca del personal de enfermería de atención directa en un hospital de segundo nivel en lca-Perú

ESTILO DE LIDERAZGO

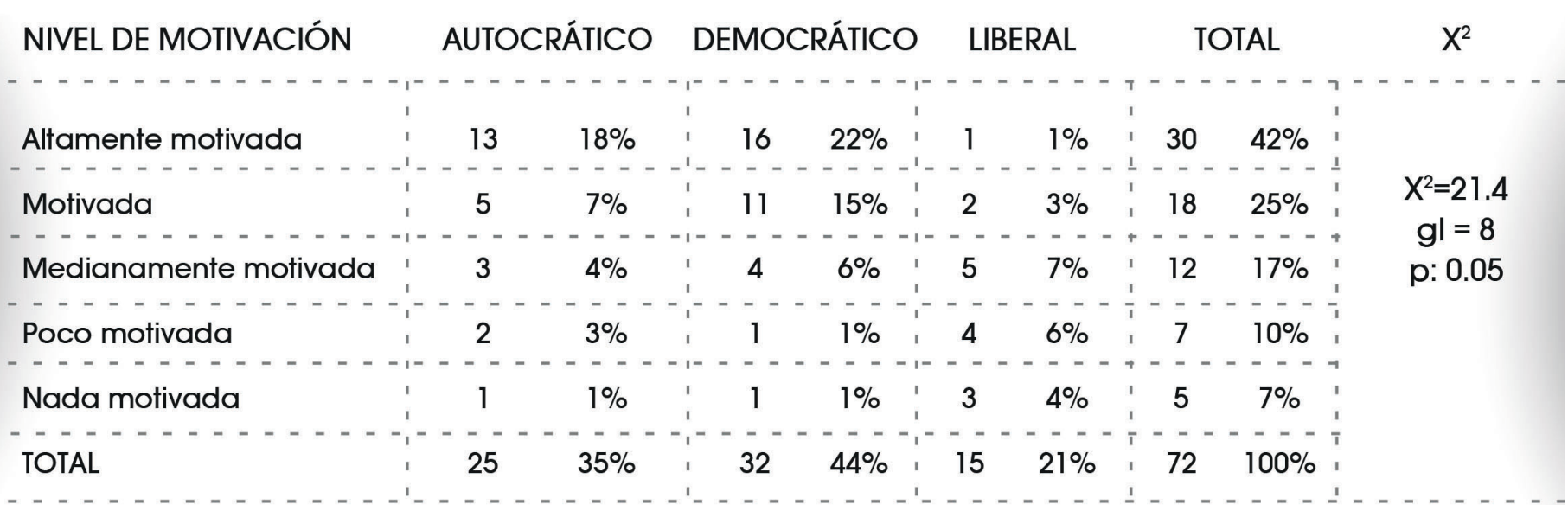

Fuente: Encuesta realizada al personal de enfermería de un hospital de segundo nivel de la ciudad de lca Perú, 2015. 


\section{DISCUSIÓN}

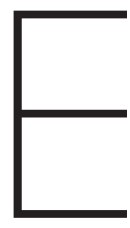

n cuanto a los datos sociodemográficos, el $57 \%$ del personal de enfermería tiene entre 41 a 56 años, el 89\% son de sexo femenino, el 47\% de estado civil casado, el 75\% tienen de 12 años a más de experiencia laboral y el 61 \% es de condición laboral nombrada, lo cual puede ser contrastado con los resultados de MarquésSánchez M. et al."16 donde las enfermeras juegan un rol de liderazgo como mediador entre las distintas subredes dentro de las unidades clínicas y esto se podría deber a que, en su mayoría, el personal de enfermería tiene más de 12 años de experiencia laboral y por su condición de nombradas les permite manejar con destreza habilidades y conductas, convirtiéndose en un factor de motivación intrínseca para beneficio de los pacientes a su cargo. Cabe destacar que el grupo estudiado, predominante, tiene experiencia laboral, seguridad y pertenece a la parte asistencial de la institución, dedicando la mayoría de su tiempo a actividades relacionadas con la mejora continua de sus conocimientos y habilidades especializadas.

Respecto a la motivación extrínseca, el 51\% del personal de enfermería se encuentra altamente motivada con los tres estilos de liderazgo, estos resultados son similares con los de Carmona-Lovera C, y Carranza-Marimon L., ${ }^{17}$ quienes manifiestan que las y los enfermeros mantienen un liderazgo continuo en el desempeño de sus intervenciones, establecen metas en un 50\%. A diferencia de Niquén-Salazar M. y Zapata-Guerrero C.18 quienes concluyen que las enfermeras asistenciales, perciben inadecuada gestión de recursos humanos y materiales, e insatisfacción con el desempeño gestor.

Esto significa que el personal de enfermería, en un alto porcentaje, se encuentra motivado extrínsecamente con el estilo de liderazgo democrático, lo cual podría deberse a que, en algunas oportunidades, la enfermera supervisora convoca a reuniones para informar sobre las actividades a realizar, consulta ideas y opiniones sobre muchas decisiones que les incumben a ambos, aceptando sus contribuciones siempre que sea posible y práctico. Sin embargo, también perciben que la enfermera supervisora asume toda la responsabilidad en la toma de deci-siones, centralizándose el poder, la toma de decisiones técnicas y funcionales del trabajo en forma autocrática. A pesar de que se entienda que van en función de los objetivos y metas del departamento de Enfermería, el personal de nivel operativo percibe que solamente la enfermera supervisora es competente y capaz de tomar decisiones importantes. Esto contrasta con los resultados del trabajo en donde, la mayoría del personal de enfermería se encuentra muy motivado extrínsecamente, por lo que se deduce que la supervisora emplea estrategias que hacen que el personal perciba que el líder es democrático en todos sus actos para lo cual se debe disponer de una buena comunicación de los objetivos a conseguir, compartir la definición del futuro de la profesión en la toma de decisiones y que les permita desarrollar con libertad sus criterios.

Respecto a la motivación intrínseca el personal de enfermería en un 42.00\% se encuentra altamente motivado. Los resultados obtenidos se pueden comparar con los de Cortés-González J. et al.."19 donde el liderazgo está asociado con la eficacia. Es decir, que el personal de enfermería, no busca una recompensa exterior, sino el placer de hacer las cosas y el trabajo porque le gusta lo que hace. Los resultados hallados permiten expresar que en su gran mayoría el personal de enfermería se siente altamente motivado con el estilo de liderazgo democrático, porque trabajan con autonomía dentro del ejercicio laboral lo cual le permite fortalecer sus competencias profesionales y realizarse profesionalmente en el servicio que labora, realizando la toma de decisiones individuales en la solución de problemas que se presentan en el servicio asignado, sin esperar beneficios ni recompensas externas.

Además, se infiere que cuanto más motivado se encuentre el personal de enfermería hacia su trabajo, según el estilo de liderazgo en la institución, mayores esfuerzos harán para desempeñar mejor 
su trabajo, por lo que el líder de hoy debe poseer un perfil muy distinto del líder de hace varias décadas, cuyo patrón se ajustaba en mayor medida al control y la supervisión.

\section{CONCLUSIONES}

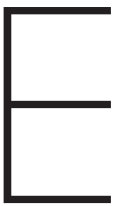
I liderazgo es el proceso de dirigir e influir en las actividades laborales de un grupo y la motivación es el estímulo que mueve a las personas a realizar determinadas acciones y persistir en ellos hasta su culminación. La supervisión es la asistencia de la realización de una actividad por parte de una persona con autoridad para ello.

\section{REFERENCIAS BIBLIOGRÁFICAS}

1. Díaz B. Liderazgo en Enfermería. México, 2007. p.3 Disponible en https://betssy3967.wordpress.com/2007/01/19/liderazgo-en-enfermeria/. (Consultado el 25 de enero del 2014).

2. Román J. Ferrández M. Liderazgo y coaching. Ed. Mc Grill. México, 2008. 200 pp.

3. Gonzales A, Pérez S. Liderazgo de enfermería-Dirección estratégica. Madrid, 2007: Disponible en: www.gestiondeenfermeria.com/liderazgo-deenfermeria-ii). (Consultado el 25 de enero del 2014).

4. Laurencio M y Cols. Liderazgo situacional: Enferm Brasil, Sao Paulo, 2002 15(1):48-52.

5. Buss M. y Cols. Formación de vínculos profesionales para el trabajo en equipo en enfermería. Enfermería global № 20. Brasil, Universidad Federa de Rio Grande, Pelotas Brasil. 2010.pp 11.

6. Díaz M. Sistema de valores y concepción cultural de los Supervisores de Hospitales sobre la investigación en enfermería. Index de Enfermería. Madrid. 2002: 39; 20 - 3

7. Bass B. Liderazgo y rendimiento más allá de las expectativas. American Psychologist. Nueva York 2004; 52 (2): 130 - 9

8. Díaz Z y Escobar D. Competencia del gerente medio de enfermería y la satisfacción de la enfermera de cuidado directo. Trabajo para optar el grado de Magíster de la Universidad de Carabobo. Caracas, 2006.

9. Goleman D. Los estilos de liderazgo En: Ricaurte E. Saval R. Club de Leones de Balboa Distrito D1- Panamá, 2017.P.4 Disponible en: www leonismoargentino.com.art/NST180. (Consultado el 12 mayo 2014).

10. Balderas M. Administración de los servicios de enfermería. Ed. Interamericana 3ํ. ed. México, 1995. 216 pp.

11. Martínez P Fernández A. Neurobiología, alimentación y vida sa/udable. Psicología. Madrid, 2002; 2(3): 55 -78.
En relación a la motivación extrínseca el personal de enfermería se encuentra altamente motivado con el estilo de liderazgo democrático y respecto a la motivación intrínseca se encuentra en gran medida motivado con el democrático más no con un estilo liberal.

\section{AGRADECIMIENTOS}

Al personal de enfermería quienes participaron en el desarrollo del instrumento de recolección de datos. A la, Dra. Lasty Balseiro Almario, por su valiosa asesoría y aportes para la culminación de la redacción del artículo científico.

12. Manrique F. Significado de ser enfermera supervisora: Una aproximación fenomenológica. Tesis para la obtención del grado de maestría en gerencia de los servicios en salud y enfermería. Universidad de Carabobo Caracas, 2009.

13. Bandura A. Teoría del aprendizaje social. Ed. SLU. Espasa Libros. Madrid 1987. 280 pp. Disponible en: stanford.edu/dept/psychology/bandura/ pajares/BanduraGarrido.PDF. (Consultado el 10 de abril 2014).

14. Maxwell J. Lo que todo Líder necesita saber. Grupo Nelson. Nashville. Tennessee, Washington, 2012.622 pp

15. Guillen C. Psicología del trabajo para relaciones laborales. Ed. McGraw Hill. Madrid, 2000, 376 pp.

16. Marqués M. El liderazgo de los profesionales de enfermería y el rendimiento organizativo: un estudio exploratorio con aplicación del análisis de redes sociales. Hispana para el análisis de redes sociales. Madrid, 2014 25. (2): 1- 140. URL disponible en: www.raco.cat/index.php/Redes/article/ viewFile/289530/377862. (Consultado el 10 de abril 2014).

17. Carmona C., Carranza L. Nivel de participación y liderazgo en la gestión del cuidado de enfermería en el nivel operativo desde la perspectiva de Diane Huber en una institución de tercer nivel de la ciudad de Cartagena de Indias. Corporación universitaria Rafael Núñez. Cartagena, 2011; 3(1):27-34. Disponible en: revistas.curnvirtual.edu.co/index.pho/cienciaysalud/article/ view/42/37. (Consultado el 12 de junio 2015)

18. Niquén M., Zapata C. Percepciones de enfermeras asistenciales sobre rol gerencial de enfermeras jefes de servicio. Hospital Nacional-Essalud. Chiclayo - Perú. Tesis para la obtención de Licenciatura. Universidad Católica Santo Toribio de Mogrovejo. Perú, 2014. 73 pp. Disponible en tesis.usat.edu.pe/.../TL_NiquenSalazarMaria_ZapataGuerrerClaudia.pdf (Consultado el día 5 de julio de 2015).

19. Cortés J. Estilos de liderazgo en jefes de servicio de enfermería. Enfermería Neurológica. México, 2012;. 12 (2): 84-94. Disponible en: www.medigraphic com/pdfs/enfneu/ene-2013/ene132f.pdf. (Consultado el día 15 de julio de 2015)

145 MMN Why Ent Neurol Vol. 16. No. 3 septiembre - diciembre 2017 